\title{
Technical solution during challenging implantation of CoreValve Evolut R 34 prosthesis in patient with bicuspid aortic valve with large annulus
}

\author{
Piotr Chodór ${ }^{1}$, Krzysztof Wilczek², Karolina Chodór-Rozwadowska ${ }^{3}$, Roman Przybylski ${ }^{4}$, Marian Zembala $^{4}$, \\ Zbigniew Kalarus ${ }^{1}$
}

\begin{abstract}
${ }^{1}$ Department of Cardiology, Congenital Heart Diseases and Electrotherapy, Medical University of Silesia in Katowice, Silesian Center for Heart Diseases in Zabrze, Poland

${ }^{2} 3^{\text {rd }}$ Department of Cardiology, Medical University of Silesia in Katowice, Silesian Center for Heart Diseases in Zabrze, Poland ${ }^{3}$ Students Scientific Society, Medical University of Silesia, Katowice, Poland

${ }^{4}$ Department of Cardiac Surgery and Transplantology, Medical University of Silesia in Katowice, Silesian Center for Heart Diseases in Zabrze, Poland
\end{abstract}

Adv Interv Cardiol 2018; 14, 4 (54): 433-434 DOI: https://doi.org/10.5114/aic.2018.79875

Bicuspid aortic valve (BiAV) is a valvular disease, diagnosed in approximately $0.5-2 \%$ of the population [1]. This anatomic abnormality is associated with a higher risk of aortic stenosis (AS) [2]. In the era of transcatheter aortic valve implantation (TAVI) adequate sizing, due to a specific morphology, is crucial [3]. In recent years, the largest size of a TAVI prosthesis - CoreValve Evolut R 34 was introduced to clinical practice, which is dedicated to 26-30 mm of the annulus diameter. An 80-year-old male patient with severe symptomatic AS, with many comorbidities and Logistic EuroSCORE $=67.68 \%$, EuroSCORE II $=32.03 \%$, was admitted to the hospital. The ultrasonographic findings showed: left ventricle ejection fraction = $28 \%$, aortic valve area $=0.7 \mathrm{~cm}^{2}$, mean transaortic gradient $=38 \mathrm{~mm} \mathrm{Hg}, V_{\max }=4.1 \mathrm{~m} / \mathrm{s}$. The diameter of the annulus in multi-slice computed tomography (MSCT) was $34.2 \mathrm{~mm}$. The patient was consulted by two Heart Teams and due to the very high operative risk, the character of the BiAV and the large annulus the patient was disqualified from surgery and TAVI. In order to implant a prosthesis with optimal oversizing (10-20\% of the perimeter of the annulus), it should have had a size of $37.7-41.1 \mathrm{~mm}$ (the perimeter of the prosthesis: $118.25-129 \mathrm{~mm}$ ). Such a large prosthesis does not exist. However due to BiAV morphology (Figure $1 \mathrm{~A}$ ) it was still possible to implant a prosthesis with a smaller size than was indicated by measurements in MSCT scans (down-sizing). In this case, a prosthesis would be secured by BiAV cusps (Figure $1 \mathrm{~B}$ ). During TAVI there was a potential risk of prosthesis displacement after implantation due to the large annulus, an occurrence of severe aortic regurgitation after balloon aortic valvuloplasty (BAV) and deformation of the implanted prosthesis. This in turn potentially might hinder retrieval of the delivery system. Moreover, there was a risk of significant paravalvular leak (PVL) after implantation. Taking into account all these considerations, we decided to perform TAVI.

The patient received the Evolut R 34 prosthesis. Due to the risk of the prosthesis falling out and BiAV morphology, the valvuloplasty was skipped. After implantation the prosthesis was deformed (Figure $1 \mathrm{C}$ ). Moreover, during the withdrawal of the delivery system, resistance was encountered. Because of the risk of valve displacement, postdilatation with a $20 \times 40 \mathrm{~mm}$ balloon was performed via a second arterial access (Figure $1 \mathrm{D}$ ). The valve was crossed with a regular $260 \mathrm{~J}$ curved wire, replaced with Confida wire over a pigtail catheter. After postdilatation the morphology of the implanted prosthesis improved and enabled successful retrieval of the delivery system. Proper shape of the valve was restored after repeated postdilatation with a $28 \times 40 \mathrm{~mm}$ balloon. Finally, there was not relevant PVL or any echocardiographic signs of cusp damage. We presented a challenging case of successful TAVI using the down-sizing approach. We demonstrated that with the favorable morphology of the BiAV it is possible to successfully implant a $34 \mathrm{~mm}$ CoreValve Evolut $\mathrm{R}$ prosthesis even if the annulus is large, beyond the recommended range. Postdilatation of the implanted valve in the presence of the delivery system is safe and effective.

\section{Corresponding author:}

Piotr Chodór MD, Department of Cardiology, Congenital Heart Diseases and Electrotherapy, Medical University of Silesia, Silesian Center for Heart Diseases, 9 M. Skłodowskiej-Curie St, 41-800 Zabrze, Poland, phone: +48 3227353 56, +48 660 442 582, e-mail: chodor_piotr@go2.pl Received: 15.08.2018, accepted: 30.08.2018. 

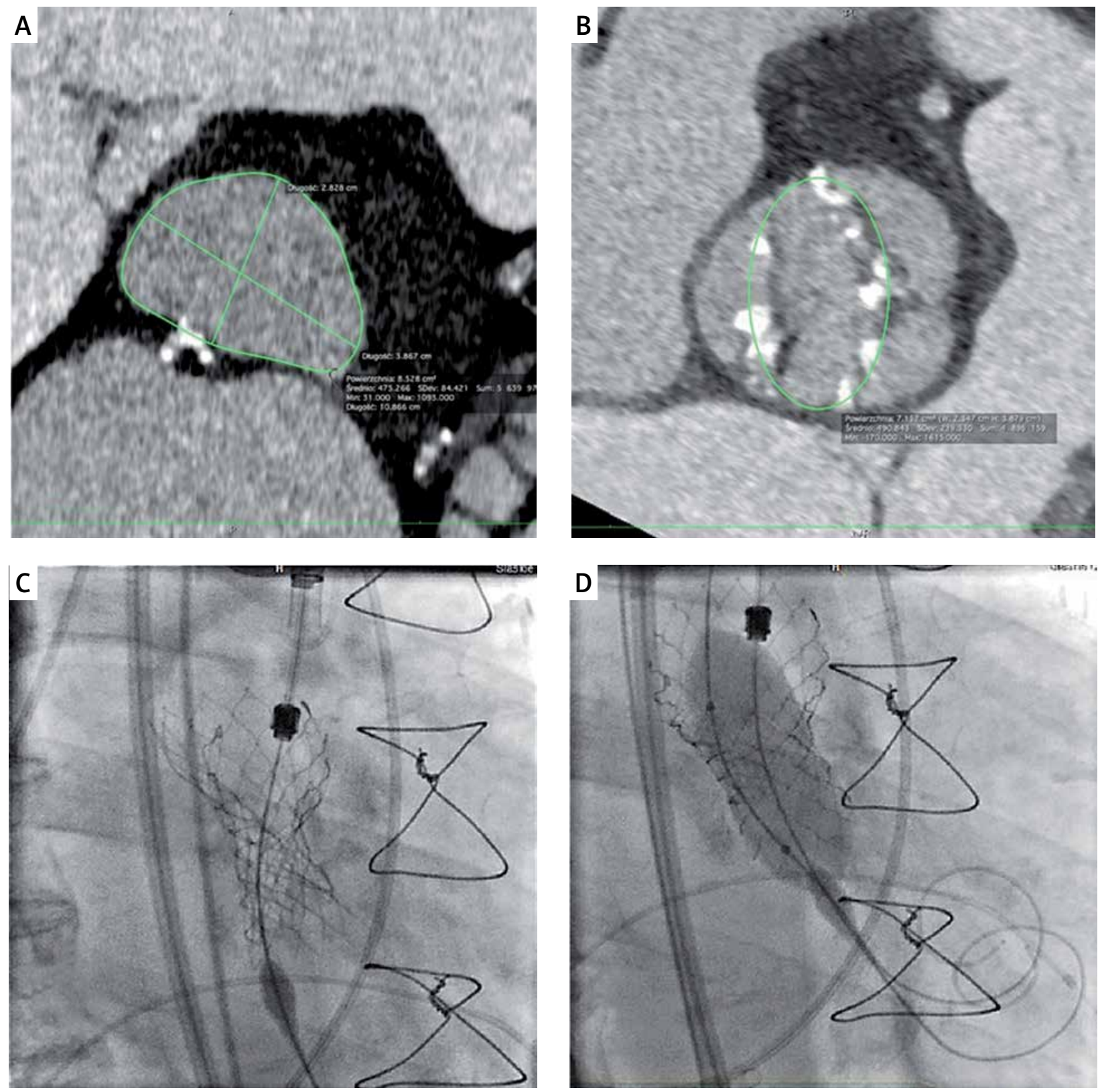

Figure 1. A - Bicuspid morphology of aortic valve in MSCT, B - implantation simulated on MSCT scan at the level of valve cusps, C - CoreValve Evolut R 34 deformed by native valve, D - BAV with balloon $20 \mathrm{~mm}$

\section{Conflict of interest}

The authors declare no conflict of interest.

\section{References}

1. Siu SC, Silversides CK. Bicuspid aortic valve disease. J Am Coll Cardiol 2010; 55: 2789-800.

2. O'Sullivan CJ, Windecker S. Implications of bicuspid aortic valves for transcatheter aortic valve implantation. Circ Cardiovasc Interv 2013; 6: 204-6.

3. Jeger R, Reuthebuch O, Fahrni G, et al. Supra-annular sizing for transcatheter valve implantation in bicuspid aortic stenosis. Adv Interv Cardiol 2018; 14: 187-90. 